\title{
Modeling spark spread option and power plant evaluation
}

\author{
Z. Li \\ Global Commodities, Bank of America, New York, USA
}

\begin{abstract}
Spark spread is an important feature in the electricity industry. The spark spread option pricing framework is presented in this paper. We start from a one-factor model and then provide a two-factor model. Closed form solution is derived. Numerical examples of spark spread option and power plant evaluation are studied. Because of the complexity of real-life constraints, we also use Monte-Carlo simulation in a more complicated example.
\end{abstract}

Keywords: spark spread, power plant evaluation, stochastic processes, hedging, real option.

\section{Introduction}

With the deregulation and liberalization of power markets in the last decade, electric markets have seen rapid changes. The power forward market is getting more and more liquid. The old cost-recovery pricing structure for electricity must adapt to market based pricing. A generator's choice can be summarized by the choice of transforming fuel into power or not. In another word, without real-life constraints, a plant can thus be thought of as a series of call options to exercise this gross profit margin, often referred to as spark spread: the difference between the prices at which power can be sold, minus the fuel costs for generating the power. The magnitude of the spark spread depends on the market prices for power and fuel as well as the heat rate, or the conversion efficiency factor of the plant:

- When the plant heat rate is lower than the market heat rate, then the unit should operate. The unit owner should buy fuel, produce power and sell to customers to take profit. The bigger the differences, the bigger the profit margin; 
- When the plant heat rate is higher than the market heat rate, then the unit should be shut down. This makes economical sense.

The unique characteristics of electricity and electricity markets present new challenges to the risk-management discipline. The most important of these are the challenges of the non-storable nature of electricity and less liquidity in power market. Our focus in developing the pricing and hedging models is on capturing the relationship of each underlying commodity, volatility of each forward as well as correlations between them.

This paper is organized as followings: Section 2 introduces the model framework. Section 3 gives some numerical example, including a power plant evaluation. Section 4 has some comments on the model.

\section{The model}

The spark spread, in its simplest form, is defined as the difference between the electricity price and the cost of generation. The payoff of the spark spread option on date $T_{e}$ is then defined as

$$
V\left(T_{e}\right)=\max \left(\varepsilon\left(F_{p}\left(T_{e}, T_{p}\right)-H \cdot F_{f}\left(T_{e}, T_{f}\right)-K\right), 0\right)
$$

where

$F_{p}(t, T)$ : the price of a power future's contract at time $t$, for delivery at time $T$, $F_{f}(t, T)$ : the price of a fuel future's contract at time $t$, for delivery at time $T$,

$T_{e}$ : Exercise date,

$H$ : Heat rate,

$K:$ Strike price,

$\varepsilon: 1$ for call and -1 for put.

Heat rate is the electricity generator's efficiency. It is defined as the number of British thermal units (BTUs) of the input fuel (measured in millions) required generating one-megawatt hour (MWH) of electricity.

\subsection{One factor normal model}

We first introduce one factor model. The spread is treated as one underline. In this case, we can use a normal model for the spread. The spark spread option is then

$$
V(0)=P\left(0, T_{e}\right)\left(\varepsilon\left(F_{p}\left(0, T_{p}\right)-F_{f}\left(0, T_{f}\right)-K\right) \Phi(\phi d)-\sigma \sqrt{T_{e}} \phi(d)\right),
$$

where

$$
d=\frac{F_{p}\left(0, T_{p}\right)-F_{f}\left(0, T_{f}\right)-K}{\sigma \sqrt{T_{e}}},
$$

and $\sigma$ is the annualized volatility of the spark spread. $\Phi(x)$ is the cumulative distribution function for the standard Gaussian distribution. $\phi(x)$ is the probability density function for the standard Gaussian distribution. 
This one factor normal model is useful when the power prices are quoted as heat rate, such as the case in ERCOT region. It is natural to assume that the spread is one underline.

\subsection{Two-factor Log-normal model}

Often we can only observe the power and fuel option market separately. So in this section we present a two-factor Log-normal model.

Consider a power future price $F(t, T)$. Assuming that we are in a probability space where information is generated by a vector-Brownian motion $W(t)$, the usual representation theorems imply that, under risk-neutral probability measure,

$$
\begin{aligned}
d F_{p}\left(t, T_{p}\right) / F_{p}\left(t, T_{p}\right) & =\sigma_{p}\left(t, T_{p}\right) d W_{p}(t) \\
d F_{f}\left(t, T_{f}\right) / F_{f}\left(t, T_{f}\right) & =\sigma_{f}\left(t, T_{f}\right) d W_{f}(t)
\end{aligned}
$$

where $\sigma_{p}\left(t, T_{p}\right)$ and $\sigma_{f}\left(t, T_{f}\right)$ are local volatilities for $F_{p}\left(t, T_{p}\right)$ and $F_{f}\left(t, T_{f}\right)$ respectively and $d W_{1}(t) d W_{2}(t)=\rho d t$.

Hence the spark spread option value is

$$
V(0, T)=P\left(0, T_{e}\right)\left(\varepsilon F_{1} A_{1}-\varepsilon F_{2} A_{2}-\varepsilon K A_{3}\right),
$$

where

$$
\begin{aligned}
A_{1} & =\int_{-\infty}^{\infty} f(x) N\left(\varepsilon d_{1}(x)\right) d x \\
A_{2} & =\int_{-\infty}^{\infty} f(x) N\left(\varepsilon d_{2}(x)\right) d x \\
A_{3} & =\int_{-\infty}^{\infty} f(x) N\left(\varepsilon d_{3}(x)\right) d x \\
f(x) & =\frac{1}{\sqrt{2 \pi}} e^{-x^{2} / 2} \\
d_{1}(x) & =\frac{\ln \frac{F_{1}}{F_{2} \exp \left(x \sigma_{2} \sqrt{T_{e}}-\frac{1}{2} \sigma_{2}^{2} T_{e}+\rho \sigma_{1} \sigma_{2} T_{e}\right)+K}+\frac{1}{2} \sigma_{1}^{2} T_{e}+\rho \sigma_{1} x \sqrt{T_{e}}}{\sqrt{\left(1-\rho^{2}\right) \sigma_{1}^{2} T_{e}}} \\
d_{2}(x) & =\frac{\ln \frac{F_{1}}{F_{2} \exp \left(x \sigma_{2} \sqrt{T_{e}}+\frac{1}{2} \sigma_{2}^{2} T_{e}\right)+K}-\frac{1}{2} \sigma_{1}^{2} T_{e}+\rho \sigma_{1} x \sqrt{T_{e}}+\rho \sigma_{1} \sigma_{2} T_{e}}{\sqrt{\left(1-\rho^{2}\right) \sigma_{1}^{2} T_{e}}} \\
d_{3}(x) & =\frac{\ln \frac{F_{1}}{F_{2} \exp \left(x \sqrt{T_{e}} \sigma_{2}-\frac{1}{2} \sigma_{2}^{2} T_{e}\right)+K}-\frac{1}{2} \sigma_{1}^{2} T_{e}+\rho \sigma_{1} x \sqrt{T_{e}}}{\sqrt{\left(1-\rho^{2}\right) \sigma_{1}^{2} T_{e}}} \\
F_{1} & =F_{p}\left(0, T_{p}\right) \\
F_{2} & =H \cdot F_{f}\left(0, T_{f}\right)
\end{aligned}
$$




$$
\begin{gathered}
\sigma_{1}=\frac{1}{T_{e}} \sqrt{\int_{0}^{T_{e}} \sigma_{p}\left(t, T_{p}\right)^{2} d t} \\
\sigma_{2}=\frac{1}{T_{e}} \sqrt{\int_{0}^{T_{e}} \sigma_{f}\left(t, T_{f}\right)^{2} d t}
\end{gathered}
$$

Since the solution is closed-form, the calculation is fast. The integral can be computed using the Gauss-Hermite quadrature method (see Press et al [2] for details).

Note $\sigma_{i}, i=1,2$ are the implied volatilities from time 0 until time $T_{e}$. In most situations the spark spread is for day ahead or real time, so the local volatilities are not critical. In the case that local volatilities structure is necessary, some other technique can be used.

As we know, a lot of new power generation use natural gas as fuel. The shape of power markets reflect this and they also display strong seasonality.

L. Anderson [1] provided an interesting two-factor mean reverting model for natural gas. Our model can easily expand with this model. We can also apply jump diffusion model to power market.

\section{Numerical example}

In this section, we give a few examples on the application of the spark spread option. The power curve is taken from PJM West region and natural gas is from Transco Zone 6 on the close of September 17th, 2007. The forward prices are from January 2008 until December 2010 and they are shown in Figure 1.

The average PJM West power prices is $\$ 78.47$ per MWH and the Transco Zone 6 gas prices is $\$ 9.87$ per MMBTU. So the market heat rate is 7950 MMBTU per KWH. Assuming a correlation of $85 \%$, Figure 2 shows the premiums with different heat rate. In reality, the variable operation and maintenance costs are often non zero. Figure 2 also shows the premiums with different heat rate, giving a variable operation and maintenance (VOM) costs of $\$ 2.5$ per MHW.
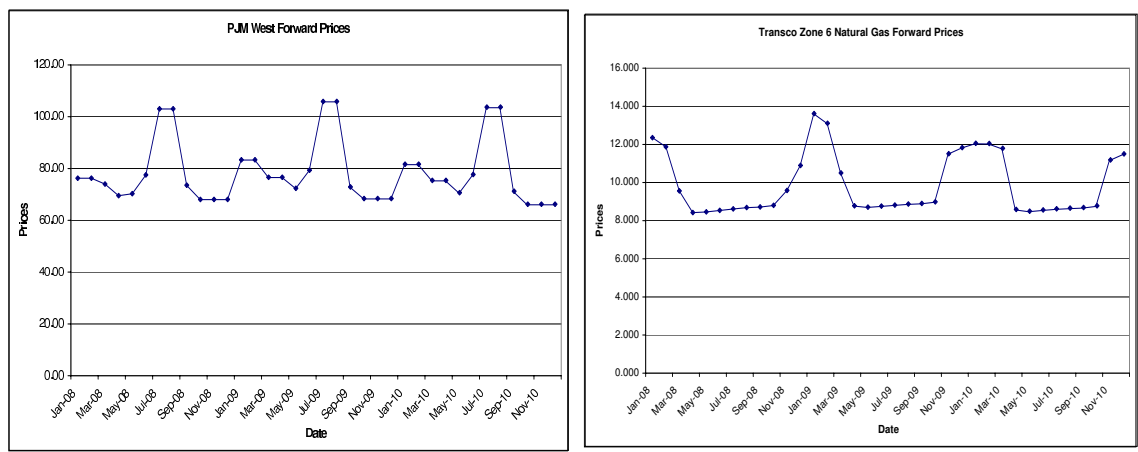

Figure 1: Power and natural gas forward curves. 


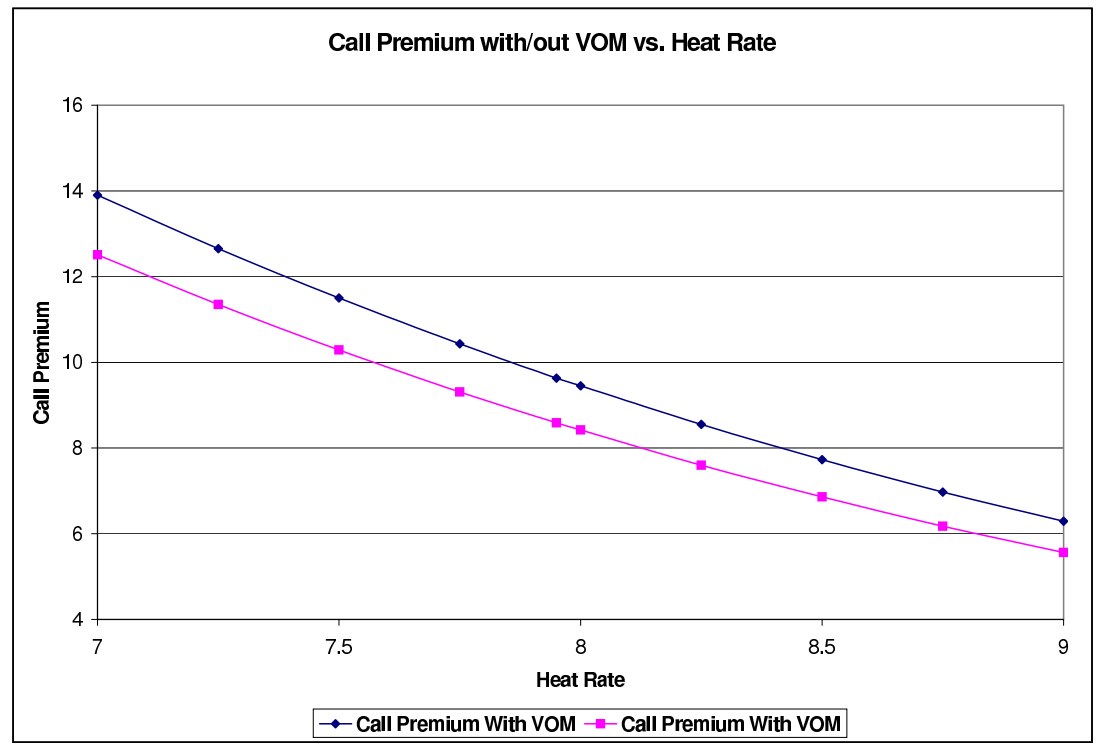

Figure 2: Call premium with/out VOM vs. heat rate.

Power plant evaluation is eventually to price a spark spread option. However, it is much more complicated. In most cases, you will see some or all of the following operation characteristics: minimum/maximum capacity, startup time, number of startups, minimum run time, startup costs, non-maintenance and variable operation maintenance, etc.

In order to properly price this kind of complicated option, we have to use MonteCarlo simulation. Suppose the power and gas future follow equations (3) and (4), we build up a two-dimensional Monte-Carlo simulation. Here we just give an example on spark spread option with VOM and startup cost.

Assuming a heat rate of 8000 MMBTU per KWH and a VOM of \$2.5 per MHW, the call without start-up cost is flat and obviously has the highest premium. Figure 3 shows the premium with different start-up cost. For comparison, the flat premium without start-up cost is also shown in the figure.

\section{Comments}

Spark spread is the primary cross-commodity transaction in electricity markets. This spread is to gauge the benefits of generating electric power with natural gas, coal and oil versus buying power directly from the electricity grid. Among all the fuels, the spark spread between electricity and natural gas is the most common spread. Options on spark spread are probably the most important cross-commodity derivatives in the electricity market.

We set up a reasonable framework to price this kind of structure. The closedform solutions are provided for the one and two-factor models. With the help of 


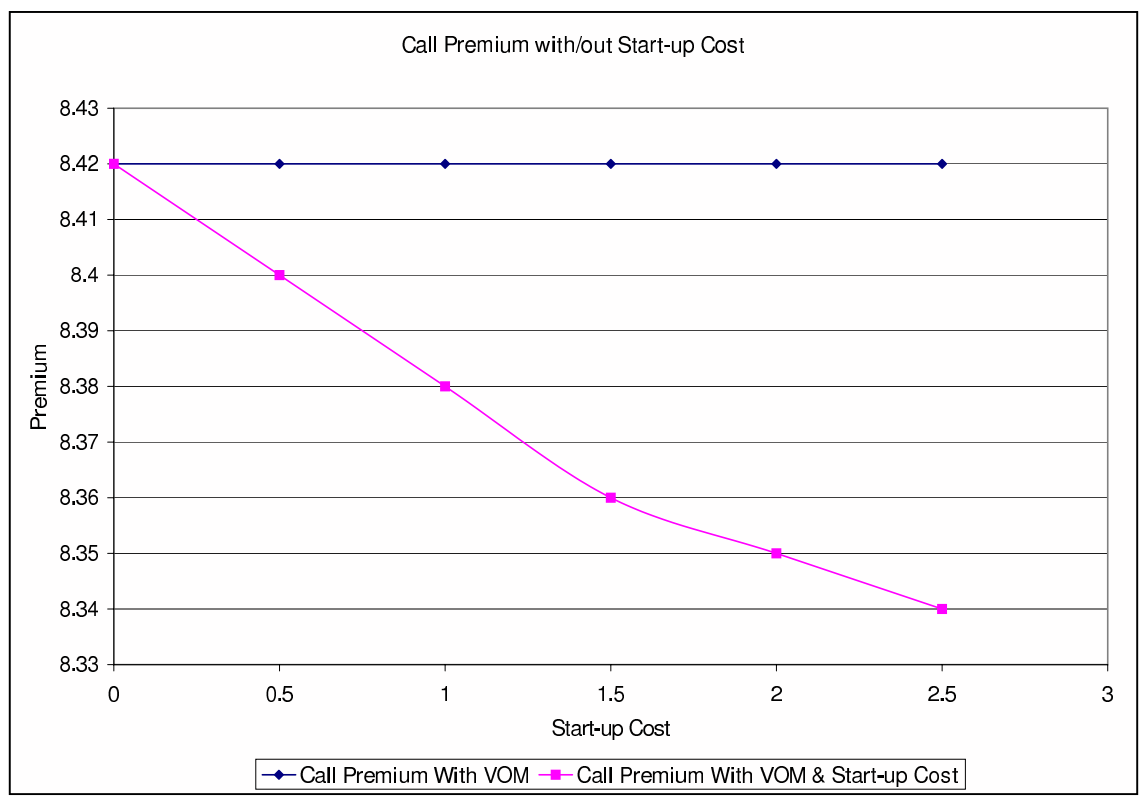

Figure 3: Call premium with/out start-up cost.

Monte-Carlo simulation, we can perform more complicated real life power plant evaluation.

There are some limitations of the models, in particular it does not incorporate "jumps" and seasonality explicitly, though volatility itself already includes this information. L. Anderson [1] provided an interesting direction to resolve these issues and his approach can be applied directly to our framework. Correlation between electricity and fuels are the input of the model, but it is hindered by the lack of market prices to calibrate them to. Our future modeling efforts will be focused in these directions. This is also dependent on the improvement of the market liquidity and more price observance.

\section{Acknowledgement}

I thank my colleagues at Global Commodities, Bank of America for their suggestions and many fruitful discussions during the course of this work.

\section{References}

[1] Andresen, L. (2007), "Notes on Gas Commodities", Bank of America internal notes.

[2] Press, H. et al. (1988), "Numerical Recipes in C", Cambridge University Press. 